\title{
Face Recognition under Variant Head Poses Using Single Frontal Gallery Image and Facial Symmetry
}

\author{
Seyed Omid Shahdi and S. A. R. Abu-Bakar
}

\begin{abstract}
Face recognition algorithms work properly only under strict conditions. There is no doubt that the system accuracy drops significantly when some of those conditions are not applied. In this paper, we take care of the most detrimental factor, variant head poses, to recognize the face. Novel method is proposed to construct the virtual view of face from a single frontal face. Instead of using the whole face, some patches are selected from only half of the face. Features are extracted via biorthogonal wavelet transform. Block of feed forward neural networks is applied to infer the mapping ratio between poses and gallery. CMU database is utilized to demonstrate the robustness of the proposed method.
\end{abstract}

Index Terms-Pose, biorthogonal wavelet transform, face recognito, human machine interaction.

\section{INTRODUCTION}

Although more than three decades of efforts have been dedicated to face recognition systems, today's systems are only reliable under strict conditions. The three most important restrictions to recognize the face in real world are illumination, expression and pose. However it has been proven that the pose is more difficult to deal with and it is well-known as a bottleneck in recognizing the face [1]-[4]. It could be shown that the extracted data from different identities show more similarity when compared to data extracted from the same identity but in different poses. If the system could recognize the face without preconditions, it will be useful for variety of applications such as security and surveillance systems and human-computer interactions.

Still there is yet another problem which has been neglected in most of previous works [5]-[12]. Specifically it is the number of face images per person used as gallery. Since in real-world it is a tedious task to provide many images from different poses of one identity in the database, it is noteworthy for the system to remain accurate by relying only on the single image per person. The frontal face is more appropriate to be used as a gallery because of the important and necessary information it provides in applications such as in a access control system as well as for biometric database (passports or ID cards).

There are different categories in the recognition of face with varying poses. In the view point of types of image, it is divided to $2 \mathrm{D}$ and $3 \mathrm{D}$. There are many works which used three dimensional images [11], [13]-[17]. These systems could obtain higher accuracy compared to 2D images.

Manuscript received April 9, 2012; revised May 10, 2012.

The authors are with the Computer Vision, Video and Image Processing (CvviP) Research Lab, Faculty of Electrical Engineering, Universiti Teknologi Malaysia, 81310 UTM Skudai, Johor, Malaysia (e-mail: shahdi@fkegraduate.utm.my, syed@fke.utm.my)
However, due to their high dimensionality of data, they are time consuming and thus not suitable for real-time applications. Another drawback of using 3D images is the huge database needed for every image at different angles. On the contrary, 2D image could be easily gathered via ordinary camera. It also does not need too much time to process the data because of its low dimensionality.

Another categorization is according to their usage of either the entire face (global methods) [5], [7], [18]-[22] or only using some patches in the face (local methods) [23][25]. Theoretically using the entire face should give the system better performance compared to using local methods. Nevertheless, since some regions of the face do not provide extra information, their inclusions do not improve the accuracy and in some situation those regions may cause misclassification. Thus, in this case local methods provide more promising results.

In the method proposed by Li et al. [1] similarity between faces is evaluated via correlations in a media subspace among different poses. Their work is also based on the patch level and in their method media subspace is constructed by Canonical Correlation Analysis in order to maximize the intra-individual correlations. However, the pose is limited to angles with which two eyes are visible.

Du et al. [2] partition the whole image into 7 fixed size patches. The virtual frontal for each patch is then estimated separately. The virtual image is constructed by integrating these virtual frontal patches. Similar to the previous methods [1], [3], the pose is confined to the orientation whereby two eyes are visible. The other problem with their method is that the fixation of patch size and its only dependency on the two centers of the eyes may cause a considerable reduction in accuracy.

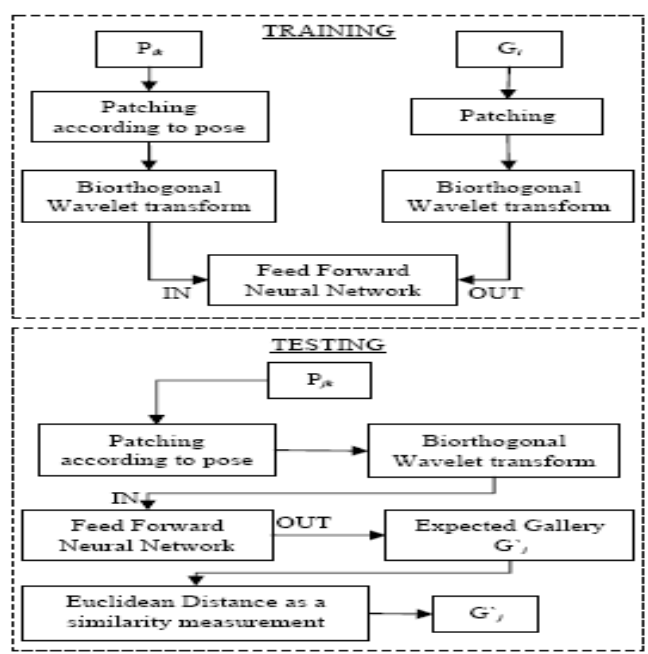

Fig. 1. Proposed method in a glimpse, where $P$ and $G$ represent pose and gallery (frontal) images respectively. Index $i$ shows the $i$ 'th person used as a training image and index $j$ shows $\mathrm{j}$ 'th person used as a probe image. Index $k$ shows the corresponding pose. 
In this paper, 2D images are used as inputs. These images are patched according to their pose. It should be noted that throughout this paper, it is assumed the pose of each face is known. Biorthogonal Wavelet transform are applied to the patches to get the second order features. By training block of feed forward neural networks, the system is learned how to construct the virtual view from frontal one. Finally, simple comparison between constructed face and gallery face defines the target identity. Fig. 1 depicts our proposed method in a glimpse. In the next section, the proposed method is overviewed. The experimental results are given in section 3. Finally in section 4, conclusion and future work are presented.

\section{Proposed Method}

In this section patching of both gallery and testing (probe) face is briefly discussed. Then biorthogonal wavelet transform is presented as a feature extractor. Feed forward neural network is the last component which is introduced in this section.

\section{A. Patching Process}

By using patches or regions of the face instead of using whole the face, the following advantages are guaranteed:

- The redundant features are removed.

- The dimension of data becomes lower and consequently the needed processing time decreased significantly.

For the frontal gallery images, seven regions are considered viz. left eye, right eye, nose tip, left mouth, right mouth, left area and right area of chin (see Fig. 2). These regions are chosen due to their importance in face recognition.

According to [25], [26] the commonly accepted assumption is that human head is bilaterally symmetrical. We take an advantage of this characteristic and therefore consider only patches on the side of the face which is fully visible. This gives us the benefit as when the direction of the face is in full or near side view; we still have reliable patches for recognition task. Therefore, four patches are extracted in the pose case, i.e. for the left side view pose, left eye, nose tip, left mouth, and left area of chin are chosen and for the right side view pose, right eye, nose tip, right mouth, and right area of chin are selected. Both gallery and pose images are patched manually. Some samples of patching the pose image are shown in Fig. 3.

\section{B. Biorthogonal Wavelet Transform}

In this paper, biorthogonal wavelet transform is adopted as a feature extractor tool. It is applied on each of the patch image.

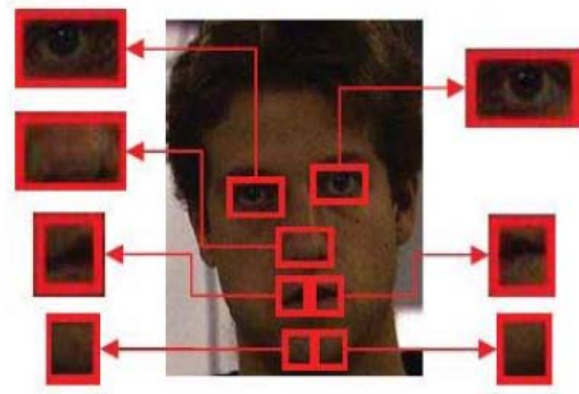

Fig. 2. Selected patches in the gallery (frontal) image
Biorthogonal wavelet is the extended version of wavelets. Instead of using the regular wavelets, the biorthogonal wavelets utilized two wavelets, one for decomposition and another one for reconstruction.

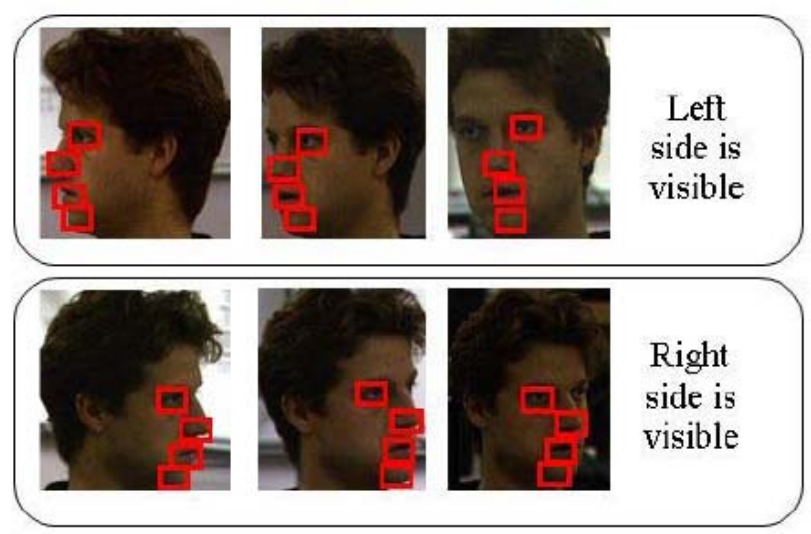

Fig. 3. Patching according to the pose (left and right are defined here as the view point of person)

The outstanding characteristic of this type of wavelets is that symmetry and precise reconstruction is feasible by using FIR filters. Biorthogonal wavelets are various in decomposition and reconstruction orders. In this paper, the biorthogonal wavelets with reconstruction order $5\left(\mathrm{~N}_{\mathrm{r}}\right)$ and also decomposition order of $5\left(\mathrm{~N}_{\mathrm{d}}\right)$ are chosen, because in this case reconstruction and decomposition functions and filters are close in value. The effective length of decomposition low pass filter and decomposition high pass filter are 9 and 11 respectively. These types of wavelets are illustrated in Fig. 4.

\section{Feed Forward Neural Network}

For the purpose of training the system in mapping the pose face to its corresponding frontal counterpart, feed forward neural network is implemented. In this stage, the features extracted from the biorthogonal wavelet are used to train the network. The input and output of the network are pose and the corresponding gallery respectively. In our work, for each element of outcome features, there is one neural network assigned.
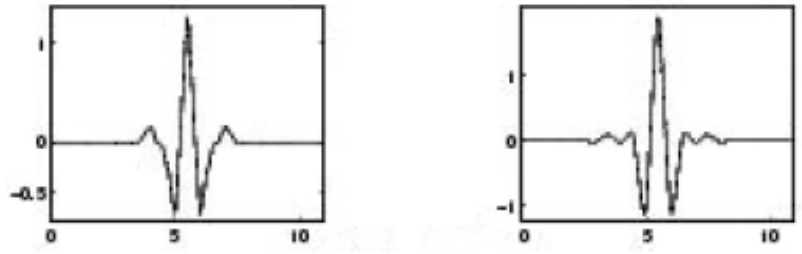

Fig. 4. Two wavelets which are used in Biorthogonal wavelet transform; the left one is used for decomposition $\left(\mathrm{N}_{\mathrm{d}}=5\right)$ and the right one is used for reconstruction $\left(\mathrm{N}_{\mathrm{r}}=5\right)$

The process of neural network learning is illustrated in Fig. 5. Block of neural networks is introduced here which contains $n$ (number of elements or wavelet features for each face) neural networks. By giving the pose as input and the corresponding gallery as target, the weights of the neural network block is updated and as a consequence, the block is trained.

The sequential training mode, learning via LevenbergMarquardt is utilized in our work. This method of learning, updates the neural network weights as follows: 


$$
w(s+1)=w(s)-\left[J^{T}(s) J(S)+\mu I\right]^{-1} J^{T}(s) e(s)
$$

where, $I$ is the identity matrix, $w(\mathrm{~s})$ is the weight at the $\mathrm{s}$ 'th iteration of the training process and $w(\mathrm{~s}+1)$ is the updated weight. The damping factor (non-negative), $\mu$, is adapted at each iteration. $J$ is the Jacobian matrix that contains first derivatives of the network errors with respect to the weights and biases, and $e$ is a vector of network errors. Mean Square Error (MSE) is employed as a performance function.

The transfer function for hidden neurons is tangent sigmoid function (2) and activation function is a linear function.

$$
\text { out }=\frac{e^{i n}-e^{-i n}}{e^{i n}+e^{-i n}}
$$

where in and out are input and output of the activation function respectively.

\section{EXPERIMENTAL RESULTS}

In testing our proposed method, we employed face images from the CMU database. We randomly divided the face images into two parts, namely training and testing.

\section{A. CMU (PIE) Database}

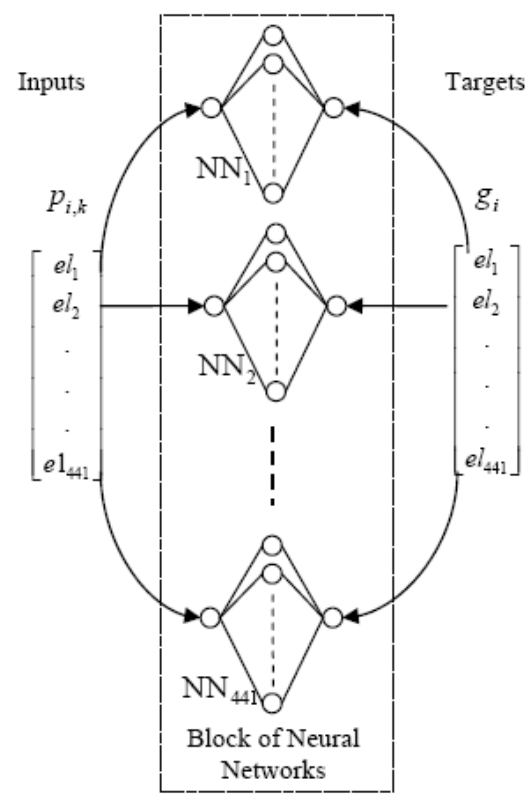

Fig. 5. Learning block of neural networks, where $w p$ and $w g$ stand for features extracted via wavelet from the pose image and features extracted via wavelet from the gallery image respectively. $e l_{n}$

shows the $\mathrm{n}$ th elements from the features. $i$ and $k$ are indicators in order for the person (subject) and the pose.

Although too many standard databases have been published for face recognition purpose, not many of them include non-frontal faces [27]. Two most common databases which have been used frequently in face recognition across varying pose domain are FERET and CMU. In comparison with CMU, FERET has some problems such as imprecise control of angle and also limitation of pose between -40 degree and 40 degree in the horizontal axis [25], [28]. CMU contains more than 40,000 facial images which were collected from 68 persons about a decade ago [29]. PIE stands for pose, illumination and expression that imply these difficulties are included in this database. To gather this database, 13 cameras were used in different angles in the CMU 3D room. Nine of these cameras were located at head height relatively from left profile to a right profile. Two other cameras were positioned higher and lower than the frontal camera, and the last two cameras were positioned in the corners [29].

For our work, the CMU faces which only include different poses are chosen with the normal condition in illumination and expression. Therefore, 68 people each one with 13 different poses are selected for our experiment. In order to further enhance the speed of the system and also to reduce complexity, all of the images were converted to grayscale and then resized from $486 \times 640$ to $82 \times 106$ by the scale of 0.17 .

\section{B. Training}

For this training phase, 34 subjects with all of their poses were used. Initially all faces were patched according to their orientations (left or right). To elaborate this, for left oriented face four patches (i.e. left eye, nose, left mouth and left chin) were selected and also for right oriented face four patches (i.e. right eye, nose, right mouth and right chin) were selected as stated in II-A. Each patch has the size of $9 \times 9$.

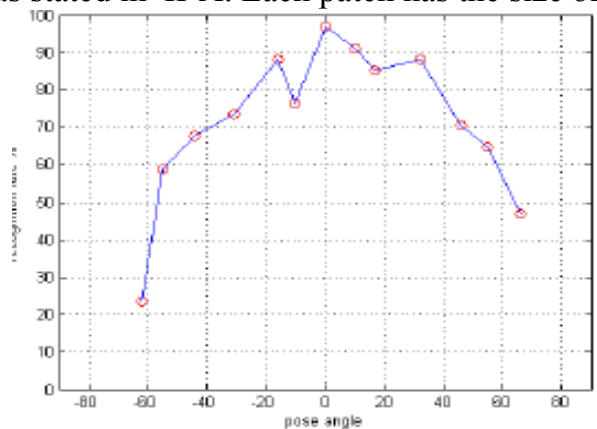

(a)

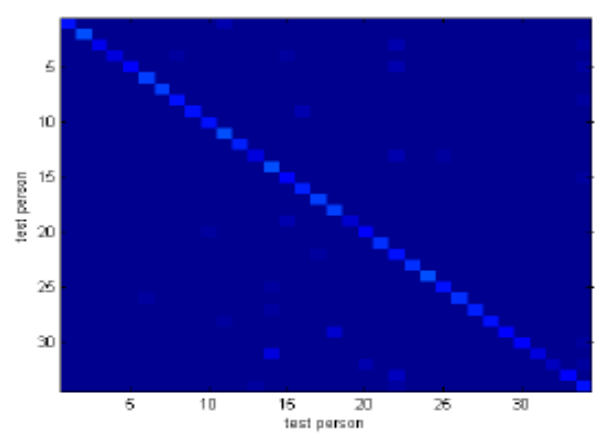

(b)

Fig. 6. Recognition results using the proposed method, a) accurate recognition rate per percent versus pose angle and b) confusion matrix of the results

Then biorthogonal wavelet transform was applied on these patches to provide second order features. By having features from both pose image and gallery image of same identity, the trained block of neural network for corresponding gallery $G$ and pose $P$ was achieved by presenting the pose as an input and gallery as an output of the neural network.

$$
G_{i}=\operatorname{Neural} \operatorname{Network} \operatorname{Block}\left(P_{i k}\right)
$$

where $G_{i}$ represents gallery of the pose, $P_{i k},(k$ th pose and 
$i$ th identity with $k=1,2, \ldots, 13 \& i=1,2, \ldots, 34)$. As mentioned in Section II_C each element has its own special neural networks. These networks have 6 hidden neurons and one neuron in input layer and also one neuron for output layer. Now each pose has its own corresponding trained block of neural networks.

These neural networks which are defined for each pose would govern the relationship between pose and frontal image of the same identity. By using these networks, frontal view of target face could be generated easily from the pose one in test section.

\section{C. $\quad$ Testing}

For the testing phase, new 34 faces were fetched to the proposed system as the probe faces. To create the virtual face (or expected gallery), the probe face is sent to its corresponding pose block of neural networks.

$$
G_{j}^{\prime}=\operatorname{Neural} \operatorname{Network} \operatorname{Block}\left(P_{j k}\right)
$$

where $G_{j}^{\prime}$ represents the expected gallery of the pose, $P_{j k}$ ( $k$ th pose and $j$ th identity which $k=1,2, \ldots, 13 \quad \&$ $j=1,2, \ldots, 34$ ).

The last step for recognition is the comparison between $G_{j}^{\prime}$ and all of the gallery face images. The decision for the correct owner of the face was made based on the Euclidean distance.

\section{Results}

The proposed method was simulated using MATLAB, running on a $2 \mathrm{GHz}$ Intel Core 2 Duo processor with $1 \mathrm{~GB}$ RAM on board. The proposed method was able to recognize the posed face with an average accuracy of $72 \%$. The recognition results are shown in Fig. 6. It is obvious that by varying the pose from the frontal to the full side view, the accuracy of face recognition system decreases.

Fig. 7 depicts the result of our proposed method in comparison with the work which has been conducted by Li et al. [1]. Note that the degree of pose orientation they used was limited to between \pm 50 degrees only.

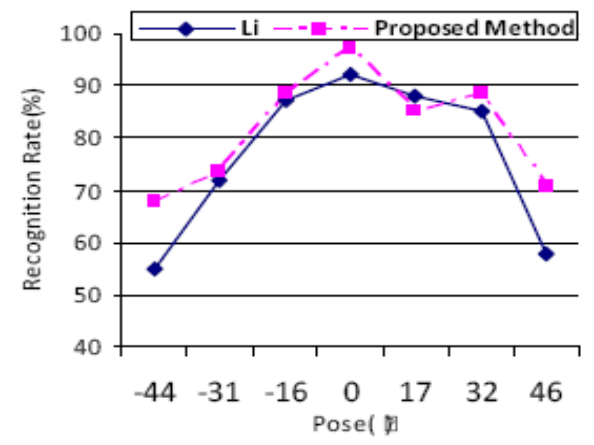

Fig. 7. Comparison of recognition rate across different poses between proposed method and $\mathrm{Li}$ et al.

\section{CONCLUSION}

A new method has been proposed in this paper to cope with the difficulties in recognizing the face of different pose. The method is very simple to implement. The results are promising even in the case of large head deviation. We are considering other features such as the structure of head, style of the hair and the color or the texture of face in our future work in an effort to increase the accuracy.

\section{ACKNOWLEDGMENT}

The authors would like to thank Universiti Teknologi Malaysia for funding and supporting this research.

\section{REFERENCES}

[1] L. Annan, S. Shiguang, C. Xilin, and G. Wen, "Maximizing intraindividual correlations for face recognition across pose differences," in Computer Vision and Pattern Recognition,CVPR 2009. IEEE Conference on, pp. 605-611, 2009.

[2] D. Shan and R. Ward, "Component-wise pose normalization for poseinvariant face recognition," in Acoustics, Speech and Signal Processing, ICASSP 2009. IEEE International Conference on, pp. 873-876, 2009.

[3] A. B. Ashraf, S. Lucey, and C. Tsuhan, "Learning patch correspondences for improved viewpoint invariant face recognition," in Computer Vision and Pattern Recognition, CVPR 2008. IEEE Conference on, pp. 1-8, 2008.

[4] T. Kanade and A. Yamada, "Multi-subregion based probabilistic approach toward pose-invariant face recognition," in Computational Intelligence in Robotics and Automation, in Proc. 2003 IEEE International Symposium on, pp. 954-959, vol. 2, 2003.

[5] P. N. Belhumeur, J. P. Hespanha, and D. J. Kriegman, "Eigenfaces vs. Fisherfaces: recognition using class specific linear projection," Pattern Analysis and Machine Intelligence, IEEE Transactions on, vol. 19 , pp. 711-720, 1997.

[6] T. F. Cootes, K. Walker, and C. J. Taylor, "View-based active appearance models," in Automatic Face and Gesture Recognition, 2000. in Proc. Fourth IEEE International Conference on, pp. 227-232, 2000.

[7] S. Lawrence, C. L. Giles, T. Ah Chung, and A. D. Back, "Face recognition: a convolutional neural-network approach," Neural Networks, IEEE Transactions on, vol. 8, pp. 98-113, 1997.

[8] S. Ting, B. C. Lovell, and C. Shaokang, "Face Recognition Robust to Head Pose from One Sample Image," in Pattern Recognition, 2006. ICPR 2006. 18th International Conference on, pp. 515-518, 2006.

[9] S. H. Chul, P. J. Hee, and K. S. Dae, "Combination of Warping Robust Elastic Graph Matching and Kernel-Based Projection Discriminant Analysis for Face Recognition," Multimedia, IEEE Transactions on, vol. 9, pp. 1125-1136, 2007.

[10] R. Singh, M. Vatsa, A. Ross, and A. Noore, "A Mosaicing Scheme for Pose-Invariant Face Recognition," Systems, Man, and Cybernetics, Part B: Cybernetics, IEEE Transactions on, vol. 37, pp. 1212-1225, 2007.

[11] Z. Xiaozheng, G. Yongsheng, and L. Maylor, "Recognizing Rotated Faces from Frontal and Side Views: An Approach toward Effective Use of Mugshot Databases," Information Forensics and Security, IEEE Transactions on, vol. 3, pp. 684-697, 2008.

[12] D. J. Beymer, "Face recognition under varying pose," in Computer Vision and Pattern Recognition, 1994. Proceedings CVPR '94. 1994 IEEE Computer Society Conference on, pp. 756-761, 1994.

[13] A. S. Georghiades, P. N. Belhumeur, and D. J. Kriegman, "From few to many: illumination cone models for face recognition under variable lighting and pose," Pattern Analysis and Machine Intelligence, IEEE Transactions on, vol. 23, pp. 643-660, 2001.

[14] M. Wai Lee and S. Ranganath, "Pose-invariant face recognition using a 3D deformable model," Pattern Recognition, vol. 36, pp. 1835-1846, 2003.

[15] Z. Xiaozheng, G. Yongsheng, and M. K. H. Leung, "Automatic Texture Synthesis for Face Recognition from Single Views," in Pattern Recognition, 2006. ICPR 2006. 18th International Conference on, pp. 1151-1154, 2006.

[16] V. Blanz and T. Vetter, "A morphable model for the synthesis of 3D faces," in Proceedings of the 26th annual conference on Computer graphics and interactive techniques, pp. 187-194, 1999.

[17] V. Blanz and T. Vetter, "Face recognition based on fitting a 3D morphable model," Pattern Analysis and Machine Intelligence, IEEE Transactions on, vol. 25, pp. 1063-1074, 2003.

[18] G. Yongsheng and M. K. H. Leung, "Face recognition using line edge map," Pattern Analysis and Machine Intelligence, IEEE Transactions on, vol. 24, pp. 764-779, 2002.

[19] Y. Gao and Y. Qi, "Robust visual similarity retrieval in single model face databases," Pattern Recognition, vol. 38, pp. 1009-1020, 2005. 
[20] M. Kirby and L. Sirovich, "Application of the Karhunen-Loeve procedure for the characterization of human faces," Pattern Analysis and Machine Intelligence, IEEE Transactions on, vol. 12, pp. 103108, 1990.

[21] M. Turk and A. Pentland, "Eigenfaces for Recognition," Journal of Cognitive Neuroscience, vol. 3, pp. 71-86, 1991.

[22] M. A. Turk and A. P. Pentland, "Face recognition using eigenfaces," in Computer Vision and Pattern Recognition, 1991. Proceedings CVPR '91, IEEE Computer Society Conference on, pp. 586-591,1991.

[23] R. Brunelli and T. Poggio, "Face recognition: features versus templates," Pattern Analysis and Machine Intelligence, IEEE Transactions on, vol. 15, pp. 1042-1052, 1993.

[24] A. Pentland, B. Moghaddam, and T. Starner, "View-based and modular eigenspaces for face recognition," in Computer Vision and Pattern Recognition, 1994. Proceedings CVPR '94, 1994 IEEE Computer Society Conference on, pp. 84-91, 1994.

[25] L. Wiskott, J. M. Fellous, N. Kuiger, and C. V. D. Malsburg, "Face recognition by elastic bunch graph matching," Pattern Analysis and Machine Intelligence, IEEE Transactions on, vol. 19, pp. 775-779, 1997.

[26] M. Bingpeng, S. Shiguang, C. Xilin, and G. Wen, "Head Yaw Estimation from Asymmetry of Facial Appearance," Systems, Man, and Cybernetics, Part B: Cybernetics, IEEE Transactions on, vol. 38, pp. 1501-1512, 2008.

[27] Y. Yagi, S. Kang, I. Kweon, H. Zha, S. Kumano, K. Otsuka, J. Yamato, E. Maeda, and Y. Sato, "Pose-Invariant Facial Expression Recognition Using Variable-Intensity Templates," in Computer Vision - ACCV 2007. vol. 4843: Springer Berlin / Heidelberg, pp. 324-334, 2007

[28] X. Zhang and Y. Gao, "Face recognition across pose: A review," Pattern Recognition, vol. 42, pp. 2876-2896, 2009.

[29] T. Sim, S. Baker, and M. Bsat, "The CMU Pose, Illumination, and Expression (PIE) database," in Automatic Face and Gesture Recognition, 2002. Proceedings. Fifth IEEE International Conference on, pp. 46-51, 2002.

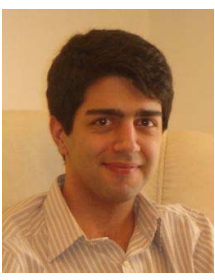

Seyed Omid Shahdi received the B.S. degree in electrical engineering from the Azad University of Yazd, Iran, in 2006 and the M.S. degree in electrical engineering from the Azad University of Qazvin, Iran, in 2009. Since 2009, he has been working toward the $\mathrm{PhD}$ degree in the computer vision, video $\&$ image processing (CvviP) lab, Faculty of electrical engineering (FKE), Universiti Teknologi Malaysia (UTM), Johor (Malaysia). His research interests include the areas of machine learning, pattern recognition, and neural networks.

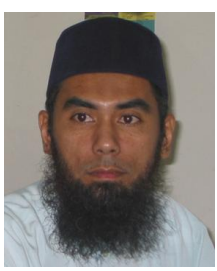

Syed A. R. Abu-Bakar received his BSc (Electrical Engineering) degree from Clarkson University in Potsdam, New York (USA), in 1990, and an MSEE degree from Georgia Tech in 1991 (USA), and the $\mathrm{PhD}$ degree from the University of Bradford, England in 1997. In 1992, he joined Universiti Teknologi Malaysia as a lecturer in the Faculty of Electrical Engineering. Currently he is an associate professor in the same faculty and holding the post of Head of Department for postgraduate studies. In 2004, he setup the Computer Vision, Video and Image Processing research lab and has become the head since then. His current research interests include image processing with applications in medical imaging, biometrics, agricultural and industrial applications, and computer vision especially in the area of security and surveillance. He has published more than 100 scientific papers both at national and international levels. He is also a senior member of IEEE. Throughout his tenure, he has obtained research grants from both government and industries amounting to a total of more than RM 1 million. 